\title{
Dust in the first quasars as a powerful probe of galaxy/BH co-evolution
}

\author{
Rosa Valiante* \\ INAF-Osservatorio Asronomico di Roma, via di Frascati 33, 00040, Monte Porzio Catone, Italy \\ E-mail: rosa.valiante@oa-roma.inaf.it
}

\section{Raffaella Schneider}

INAF-Osservatorio Asronomico di Roma, via di Frascati 33, 00040, Monte Porzio Catone, Italy

E-mail: raffaella.schneidereoa-roma.inaf.it

The origin of the large dust masses $\left(>10^{8} M_{\odot}\right)$ revealed by millimetre $(\mathrm{mm})$ and sub-mm observations of $z>5$ quasars is still hotly debated and interpreting/understanding the strong connection between the formation and growth of the first quasars and their host galaxies represents a challenge for both models and observations.

By means of the semi-analytical model GAMETE/QSOdust (Valiante et al. 2011), we reconstruct the hierarchical build-up of high redshift QSOs and their host galaxies in a cosmological context. In addition, the chemical properties of the host galaxies, namely the mass of gas, stars, metals and dust are consistently followed in the model.

As a pilot study, we have applied the model to SDSS J1148+5152 (J1148) at $z=6.4$, using this source as a template for the general population of high- $z$ QSOs. We show how dust formation and evolution may provide insights on the physical processes driving and regulating the co-evolution of quasars and their hosts in the early Universe: the dust mass produced by stars depends on the stellar initial mass function (IMF) and on the shape of the star formation history (SFH), which is controlled by the evolution of the gas content through mergers and outflows driven by Supernovae $(\mathrm{SNe})$ and by the active galactic nucleus $(\mathrm{AGN})$; the total mass of metals is a tracer of the integrated star formation history (total stellar mass) and it is sensitive to the strength and frequencies of merger-driven starbursts, metal-rich outflows, as well as to the detrimental effect of accretion onto grains in the dense interstellar medium. The above findings, complemented by a detailed analysis of the spectral energy distribution (SED) of J1148 by means of a radiative transfer code, point to an evolutionary scenario where the nuclear $\mathrm{BH}$ and the stellar bulge grow hand-in-hand by means of merger-driven gas inflows; when the $\mathrm{BH}$ mass exceeds $\sim 10^{8} M_{\odot}$, a strong $\mathrm{AGN}$ driven wind drives a powerful outflow that is able to clear-up the ISM of its dust and gas content. This is the stage when the bright quasars outshine their host galaxies. We show that this scenario naturally predicts the formation of a stellar bulge with a mass of $4 \times 10^{11} M_{\odot}$, that exceeds by more than one order of magnitude the mass allowed by dynamical constraints in the inner $2.5 \mathrm{kpc}$ of the host galaxy.

We have extended the analysis to a larger sample of quasars observed between redshift $z=5$ and $z=6.4$. We find that the best-fit scenario proposed to explain the formation and chemical evolution of J1148 and its host galaxy can explain the properties of the first QSOs in general.

The Life Cycle of Dust in the Universe: Observations, Theory, and Laboratory Experiments - LCDU 2013, 18-22 November 2013

Taipei, Taiwan

${ }^{*}$ Speaker. 


\section{Introduction}

The observation of luminous quasars ( $>10^{47} \mathrm{erg} / \mathrm{s}$ ) up to $z \sim 7$ imply black hole (BH) masses of $>10^{9} M_{\odot}$. Such super massive BHs have a relatively short time to grow by continuous gas accretion and merging with other BHs. At the Eddington rate, a seed BH of $>10^{2}-10^{3} M_{\odot}$ has to grow for at least $0.6 \mathrm{Gyr}$ to reach $\sim 10^{9} M_{\odot}$ and must have formed when the Universe was relatively young $(<1 \mathrm{Gyr})$.

Local scaling relations suggest that the central BHs and their host galaxies co-evolve. As an example, in left panel of Fig. 1 we show the observed relation between the BH mass, $M_{\mathrm{BH}}$ and stellar mass, $M_{\text {star. }}$. In AGN-selected samples the mean $M_{\mathrm{BH}} / M_{\text {star }}$ ratio seems to evolve to larger values at higher redshifts: a factor of $\sim 10$ lower $M_{\text {star }}$ than expected from the local relation is estimated for $z>5$ quasars, suggesting a faster evolution of their BHs with respect to their local counterparts. However, at such high redshifts, $M_{\text {star }}$ is inferred as the difference between the dynamical mass, $M_{\mathrm{dyn}}$, and molecular gas mass, $M_{\mathrm{H}_{2}}$, through the detection of $\mathrm{CO}$ emission lines. The major sources of uncertainties in this computation are the $\mathrm{CO}-$ to $-\mathrm{H}_{2}$ conversion factor, $\alpha_{\mathrm{CO}}$, the radius, $R$, and inclination angle, $i$, of the molecular gas disc traced by the $\mathrm{CO}$ emitting regions. We compute $M_{\mathrm{H}_{2}}$ and $M_{\mathrm{dyn}}$ assuming $\alpha_{\mathrm{CO}}=0.8 M_{\odot} /\left(\mathrm{K} \mathrm{km} \mathrm{s}^{-1} \mathrm{pc}^{2}\right), R \sim 2.5 \mathrm{kpc}, i=65^{\circ}$ for $\mathrm{J} 1148$ and $i=40^{\circ}$ for other $z>5$ quasars. The results are shown in Fig. 1, [1], [2].

Finally, large dust masses, $M_{\text {dust }}>10^{8} M_{\odot}$, are inferred from the observed rest-frame far-IR (FIR) luminosities. In the right panel of Fig. 1 we show $M_{\text {dust }}$ as a function of $M_{\text {star }}$ at $z>5$ from [2]. The shaded region represents the maximum mass of dust produced by AGB stars and $\mathrm{SNe}$ assuming a (maximum) IMF-averaged dust yield of $4.7 \times 10^{-4}-7.4 \times 10^{-3}$ (see next Section). It is clear that for most of these QSOs stellar sources are not enough to explain the observed dust masses.
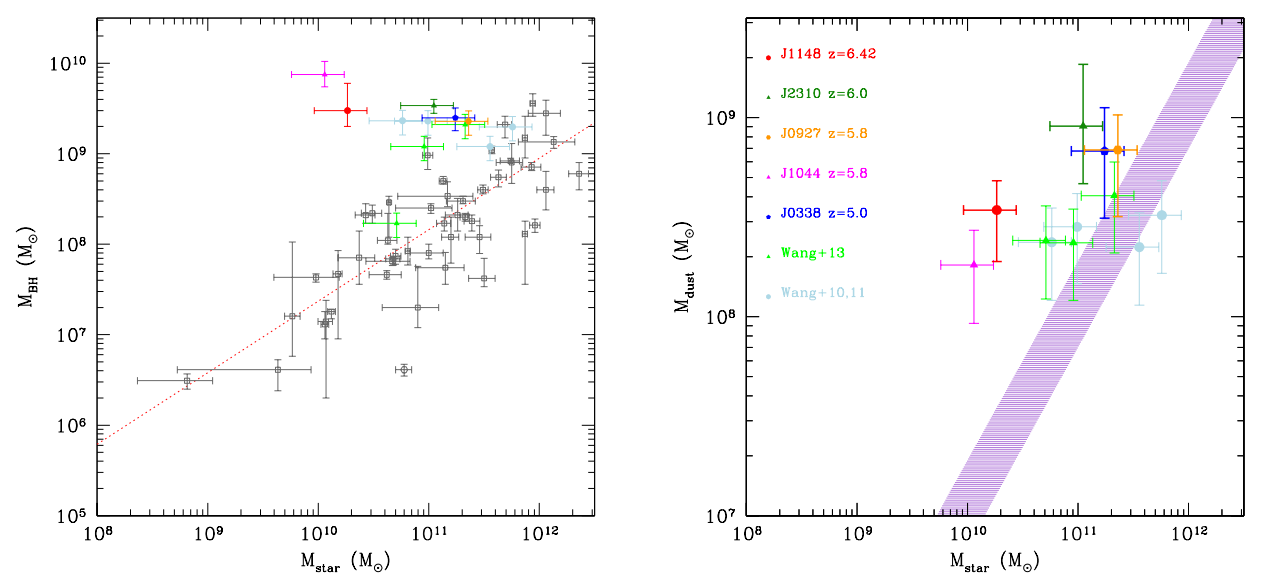

Figure 1: Left panel: The observed BH-stellar mass relation. Local galaxies are represented by grey data points with the empirical fit (dotted line) by [4]. Coloured data show a sample of $z>5$ quasars (see [2] for details). Right panel: the mass of dust as a function of the stellar mass inferred from the observations. The shaded region shows the maximum dust mass produced by AGB stars and SNe (see text). 


\section{Sources of dust}

The origin and nature of dust in the early Universe is still matter of debate. Among the stellar sources, the most efficient dust grains factories are the cool and dense atmosphere of Asymptotic Giant Branch (AGB) stars and the expanding ejecta of core collapse SNe. Progenitors of AGB stars are intermediate mass stars (IMS), in the range $(1-8) M_{\odot}$ while core collapse $\mathrm{SNe}$ are the final stage of more massive stars, $>8 M_{\odot}$.

In the left panel of Fig. 2 we show the mass of dust produced by IMS as a function of the mass of the progenitor star at solar metallicity $Z=Z_{\odot}$ from [5] and at $Z=5 \times 10^{-2} Z_{\odot}$ from [5] and [6]. The differences between these two models are discussed in a series papers in which the authors couple, for the first time, stellar evolutionary models with the model of dust production (see [2] and [3]).

In the right panel of Fig. 2, we show the mass of dust produced by SNe as a function of the progenitor stellar mass, as predicted by [7] for $Z=Z_{\odot}$ and $Z=0.1 Z_{\odot}$, before and after the passage of the reverse shock that can destroy part of the dust. Theoretical yields are compared to observations of warm (light blue points) and cold dust (blue and red points) in SNe and SN remnants. It is
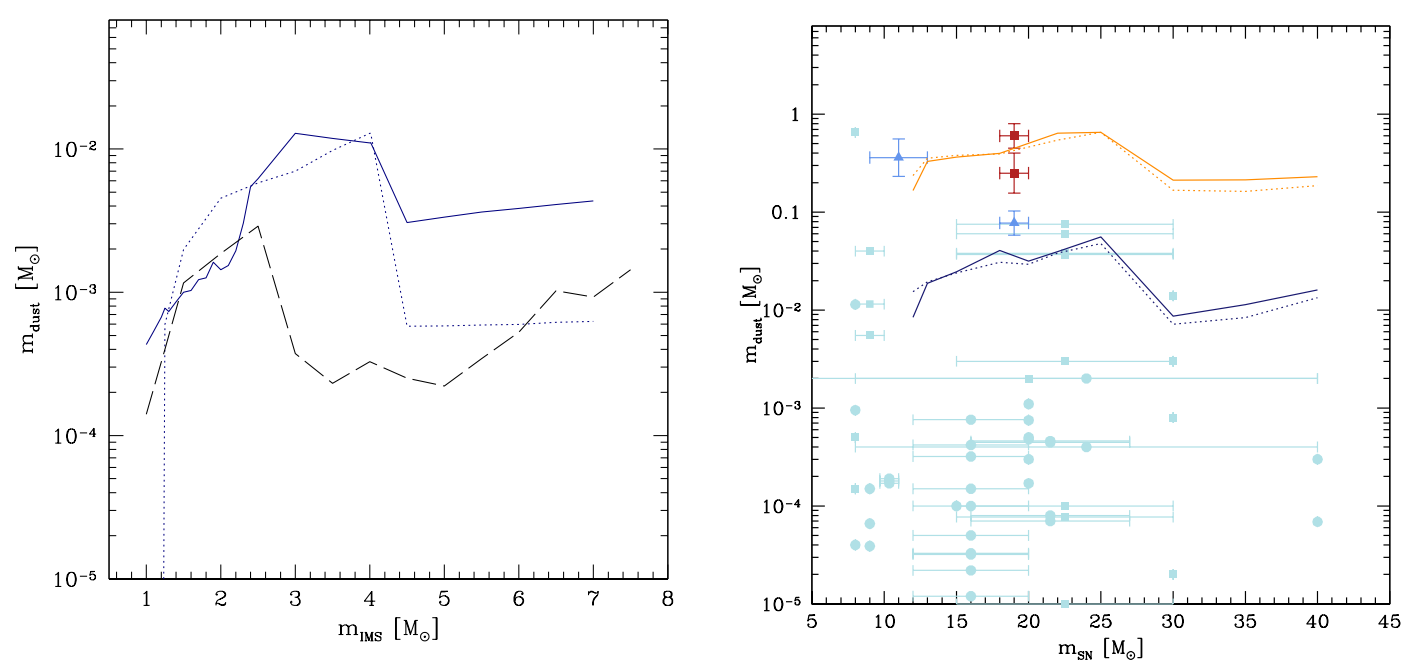

Figure 2: Total mass of dust produced by AGB stars (left panel) and SNe (right panel) as a function of the stellar progenitor mass for $Z=Z_{\odot}$ (solid lines) and $Z=5 \times 10^{-2} Z_{\odot}$ (dotted lines). The dashed line in the left panel is for AGB-dust at $Z=5 \times 10^{-2} Z_{\odot}$ from [6]. Data points in the right panel are observations of SNe and SN remnants and the upper (lower) set of lines show theoretical SNe yields with no reverse shock (with reverse shock, see [9] for details).

clear that the relative importance of AGB stars and SNe as dust factories depends on the destruction efficiency of the SN reverse shock, that is still poorly constrained by the observations.

In addition, [10] showed that the relative contribution of AGB stars and SNe to dust production depends more on the SFH and IMF than on cosmic time. For a Larson standard IMF $\left(m_{c h}=0.35\right.$ $\left.M_{\odot},[8]\right)$ and a SFH dominated by a single burst, AGB stars can dominate dust production in $\approx 150 \mathrm{Myr}$, and thus can play a non-negligible role in dust enrichment even at $z>6$.

In Fig. 3 we show the integrated stellar yields, namely the total amount of dust and metals produced by AGB stars and SNe with solar metallicity progenitors per unit stellar mass formed. 
We use AGB stars dust yields from [5] and SNe dust yields computed by [7] with no reverse shock destruction (right panel) and with moderate reverse shock destruction (left panel). The thick shaded curves account for the dependence of the yield on the assumed IMF, going from a Larson standard $\left(m_{c h}=0.35 M_{\odot}\right)$ to a top-heavy $\left(m_{c h}=5 M_{\odot}\right)$ IMF. A maximum dust yield of $Y_{d}=7.8 \times 10^{-4}$ $\left(7.4 \times 10^{-3}\right)$ is obtained at $900 \mathrm{Myr}$ after the burst for a top-heavy IMF adopting SN yields in the reverse (no reverse) shock model. For a standard IMF, these numbers are reduced to $4.7 \times 10^{-4}$ $\left(3.0 \times 10^{-3}\right)$.
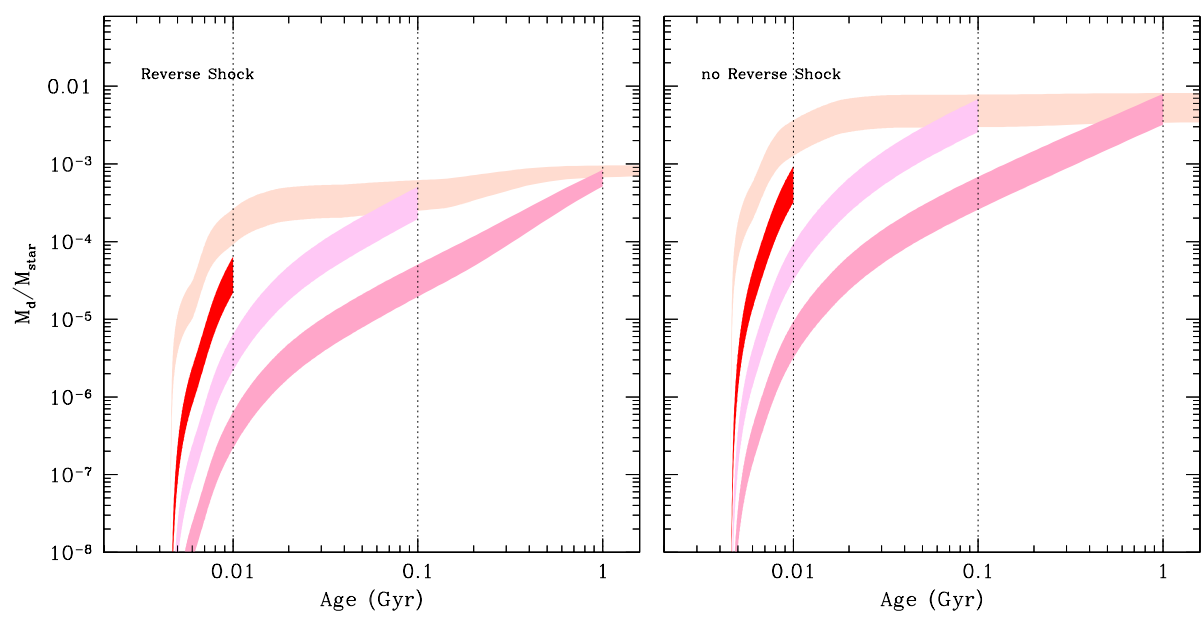

Figure 3: The integrated mass of dust produced by stars per unit stellar mass formed. Thick shaded regions represent the range obtained with a standard and a top-heavy IMF. Dust yields from SNe are computed assuming no reverse shock destruction (upper panel) or moderate destruction by the reverse shock (lower panel). The different curves are for $M_{\text {star }}$ formed in an instantaneous burst of star formation (SF) at $t=0$ or at a continuous and constant rate over a timescale of $t_{\mathrm{SF}}=0.01,0.1,1 \mathrm{Gyr}$.

As an example, an upper limit to the stellar mass of the QSO J1148 at $z=6.4$ can be estimated as the difference between the dynamical and gas masses: $M_{\mathrm{dyn}}-M_{\mathrm{H}_{2}}$, yielding $M_{\mathrm{star}}<2 \times 10^{10} M_{\odot}$; at the star formation rate inferred from the FIR luminosity of $\sim(1-2) \times 10^{3} M_{\odot} / \mathrm{yr}$, this stellar mass would be produced in a relatively short time, $\sim(10-20)$ Myr. Using the results shown in Fig. 3, we find that the maximum dust mass produced by stellar sources is $M_{\text {dust }}=4.8 \times 10^{6} M_{\odot}$ $\left(6.6 \times 10^{7} M_{\odot}\right)$, for stars forming in a single burst with a top-heavy IMF and reverse (no reverse) shock models for SN yields. Hence, even assuming a maximally efficient stellar dust production, with no grain destruction by interstellar shocks, we find that an additional source of dust is required.

\section{The evolution of high- $z$ QSO}

To build a consistent evolutionary scenario which explain the observed properties of high redshift quasars, we have developed the semi-analytical model for the formation and evolution of high redshift quasars, GAMETE/QSODUST, described in [1] and [2]. This code consistently follows the hierarchical assembly and merger history of the host galaxy and its central BH as well as the formation and evolution of the mass of gas, stars, metals and dust in the ISM. As a pilot 
study we have investigated the case of J1148, at $z=6.4$, exploring different SFHs and IMFs for the stellar populations in the host galaxy. In Fig. 4, we show the results obtained adopting two alternative scenarios: the blue lines refer to a model where stars form in efficient merger-driven bursts with a standard IMF; in this case, a large star formation rate of $10^{3} M_{\odot} / y r$ is found at the final redshift and the growth of the SMBH is accompanied by the growth of a large stellar bulge, with a mass exceeding the upper limits inferred by dynamical constraints and $\mathrm{CO}$ observations (red point in the middle panel). This large stellar bulge is required to explain the observed dust mass. In the second scenario (yellow lines) a lower efficiency of star formation is adopted and, as a result, the final mass of the stellar bulge is consistent with observational constraints. Yet, in this case the star formation rate at $z=6.4$ is $<100 M_{\odot} / \mathrm{yr}$ and a top-heavy IMF is required to reproduce the observed dust mass.
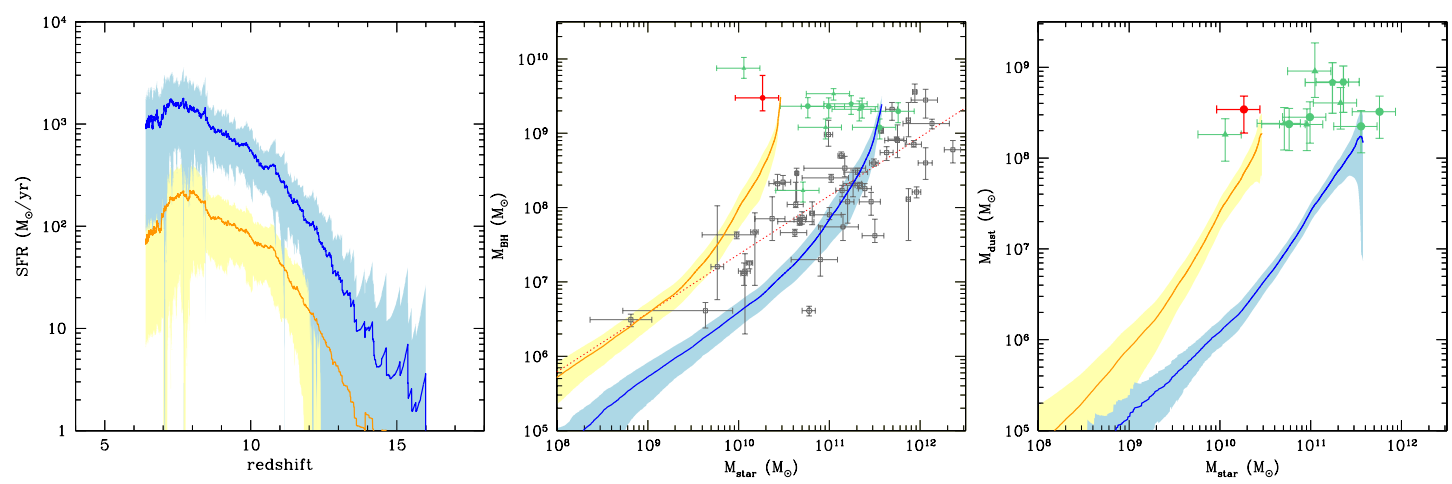

Figure 4: Model predictions for J1148 assuming two different evolutionary scenarios: high (low) star formation efficiency with a standard (top-heavy) IMF shown with the blue (yellow) shaded regions. The solid lines correspond to averages over 50 different merger histories of the QSO host galaxy, with the shaded region indicating the 1- $\sigma$ dispersion. Left, central, and right panels show the SFH and the evolution in the $M_{\mathrm{BH}}-M_{\text {star }}$ and in the $M_{\text {dust }}-M_{\text {star }}$ planes. The red points correspond to $\mathrm{J} 1148$, the green points represent a sample of QSOs at $z>5$ and the grey points indicate local galaxies.

In both scenarios, the dust mass budget is determined by the interplay between accretion in molecular clouds, destruction in the diffuse medium and ejection due to SN- and AGN-driven winds. Stellar sources seed the ISM with the first grains and gas-phase metals, but the dominant dust mass is produced by grain growth in dense molecular clouds.

We have tested these alternative scenarios using a radiative transfer model to predict the resulting spectral energy distribution (SED) of J1148, [13]. We follow the transfer of radiation produced by the AGN and by stellar sources through the dusty ISM of the host galaxy. Comparing the model predictions with the observational data, including the recently published Herschel detections, we find that the SED of J1148 points to an evolutionary scenario where $\approx 60 \%$ of the observed FIR luminosity is due to dust heating by the central AGN; heating due to stellar radiation provides a necessary but not dominant component to the emission at $(20-60) \mu \mathrm{m}$. We also find that the required stellar luminosity can only be achieved if the host galaxy is able to substain a star formation rate of $\approx 10^{3} M_{\odot} / y r$ at $6.4<z<8$ through efficient merger-driven bursts. Hence, among the two scenarios presented in Fig.4, the favoured one is the one where stars form according to a normal IMF but with large efficiencies, [1]. 


\section{Conclusions}

Understanding the origin of the large dust masses observed in QSOs host galaxies at $z>5$ is tightly connected to the nature and formation rate of the first stellar populations. Our study underlines the importance of modelling the chemical evolution of the ISM with dust in a cosmological framework in which the BH-host galaxy co-evolution is consistently followed. Using one of the most distant quasar observed, J1148, as a proxy for the general population of high- $z$ QSOs, we find that the observed properties of the host galaxy point to an evolutionary scenario where efficient merger-driven gas inflows drive an early co-evolution of the nuclear $\mathrm{BH}$ and the associated stellar bulge; when the $\mathrm{BH}$ reaches a threshold mass of $10^{8} \mathrm{M}_{\odot}$, AGN-driven wind produces large outflows that rapidly deplete the gas and dust content of the host galaxy. This sets the transition from the starburst-dominated to the bright QSO phase. Within this scenario, stars form with a standard IMF resulting in a large stellar bulge at $z=6.4$; this is one order of magnitude larger than the upper limit $M_{\mathrm{dyn}}-M_{\mathrm{H}_{2}}$ inferred from CO observations in the innermost $2.5 \mathrm{kpc}$. This leads us to the conclusion that we are either missing a fraction of the host galaxy stars or a substantial correction/revision to the method used to compute stellar masses of high- $z$ QSOs hosts must be considered. An extensive analysis of this stellar mass crisis will be discussed in [2] where we extend our analysis to a large sample of quasars between $z=5$ and $z=6.4$.

\section{References}

[1] R. Valiante et al., The origin of the dust in high-redshift quasars: the case of SDSS J1148+5251 MNRAS 416 (2011) 1916 [arXiv:1106.1418]

[2] R. Valiante et al., The stellar mass crisis in high-redshift QSOs host galaxies (2014), submitted

[3] F. Dell'Agli et al., Dust from AGBs: from carbon to silicates, in proceedings of Life Cycle of Dust in the Universe conference, POS (LCDU2013) 092

[4] E. Sani et al. The Spitzer/IRAC view of black hole-bulge scaling relations MNRAS 413 (2011) 1479 [arXiv:1012.3073]

[5] S. Zhukovska et al., Evolution of interstellar dust and stardust in the solar neighbourhood MNRAS 479 (2008) 453 [arXiv:0706.1155]

[6] P. Ventura et al. Dust from AGBs: relevant factors and modelling uncertainties MNRAS accepted (2014)

[7] S. Bianchi \& R. Schneider, Dust formation and survival in supernova ejecta MNRAS 378 (2007) 973 [arXiv:0704.0586]

[8] R.B. Larson, Early star formation and the evolution of the stellar initial mass function in galaxies MNRAS 301 (1998) 569

[9] R. Schneider et al. The AGB dust production rate in the Magellanic Clouds (2014), submitted

[10] R. Valiante et al. Stellar sources of dust in the high-redshift Universe MNRAS 397 (2009) 1661 [arXiv:0905.1691]

[11] R. Maiolino et al. Evidence of strong quasar feedback in the early Universe MNRAS L425 (2012) L66 [arXiv:1204.2904]

[12] R. Valiante et al. Quasar feedback in the early Universe: the case of SDSS J1148+5251 MNRAS L427 (2012) L60 [arXiv:1205.3488]

[13] S. Schneider et al. The spectral energy distribution of $z \sim 6$ quasars: the case of SDSS J1148+5251 (2014), submitted [arXiv:1402.2279] 